\title{
Intercostal nerve conduction and posterior rhizotomy in the diagnosis and treatment of thoracic radiculopathy
}

\author{
E. RALPH JOHNSON ${ }^{1}$, JOE POWELL, JAMES CALDWELL, \\ AND CHARLES CRANE
}

From Baylor University Medical Center, Dallas, Texas, U.S.A.

SYNOPSIS Intercostal nerve conduction study has proved to be an accurate technique in diagnosis of thoracic radiculopathy in 161 patients, 80 of whom had subsequent posterior rhizotomy with relief of pain in $81 \%$ of those undergoing surgery. The only significant complication of intercostal nerve conduction study is an $8.8 \%$ incidence of pneumothorax.

The development of the intercostal nerve motor conduction study, a new electrodiagnostic technique for establishing a definitive diagnosis in thoracic radiculopathy, has previously been reported by Caldwell et al. (1968). The purpose of this paper is to report five years' experience with this technique and the results of subsequent surgery at Baylor University Medical Center, Dallas, Texas.

The first 11 primary divisions of the 12 thoracic nerves are described as intercostal nerves, with the 12 th termed the subcostal nerve. The intercostal nerves are distributed chiefly to the parietes of the thorax and abdomen. The 1st thoracic nerve divides into two parts, one of which enters the roots of the brachial plexus, the other becoming the first intercostal nerve. The second through the 6th thoracic nerves and the intercostal section of the 1st thoracic nerve segmentally innervate the thoracic wall and the intercostal muscles. The 7th through the 12th innervate the thorax and abdominal wall and intercostal muscles.

Compression, inflammation, scarring, or traumatic irritation of the thoracic nerve roots or intercostal nerves can produce chest or abdominal pain simulating cardiac, pulmonary, or abdominal disease. Previously the absence of

\footnotetext{
1 Address for reprints: Department of Physical Medicine and Rehabilitation, University of Texas Southwestern Medical School, 5323 Harry Hines Building, Dallas, Texas, U.S.A., 75235.
}

objective clinical signs or definitive diagnostic v techniques made the diagnosis of thoracic roo $\omega$ pain one of exclusion. In some cases thoracis myelography revealed a defect which establishe the pathology. However, the majority of patients with symptoms of thoracic radiculopathy have negative myelograms. Frequently multiple diago nostic and surgical procedures were done in api $\overrightarrow{0}$ attempt to remove the pain with no specifie benefit. Posterior rhizotomy is a pain-relieving procedure (White, 1965). Neurosurgeons, however, are reluctant to operate when the diagnosis and level of involvement are unclear. Denervation potentials found in electromyographic examination of the thoracic paraspinous muscles might suggest the diagnosis but do not establish a root level. Intercostal motor nerve conduction study is a definitive electrodiagnostic technique in establishing the diagnosis and level of thoracic radiculopathy.

\section{METHODS}

The patient is placed under light anaesthesia and is then positioned to lie on the uninvolved side. Recording electromyographic needle electrodes are placed in the desired interspace in the mid-axillary line. Two $1 \frac{1}{2}$ inch Meditron insulated monopolar electromyographic needles for stimulating are of inserted in the corresponding interspace as near the $N$ spinal column as technically possible. The electrode tips are placed adjacent to the intercostal nerve with 
the negative stimulation electrode located distal to the spinal cord. A ground electrode is placed between the stimulating and recording needles. Both a Meditron Model 201-AD and TECA Model B-2 Electromyograph were used in this study. As the stimulus voltage is increased, the recording and stimulating electrodes are adjusted to give a maximal response, which is recorded by Polaroid photography. Time between stimulus and muscle response is recorded in milliseconds and the photographs screened for abnormal dispersion in the muscle response. The same procedure is conducted on each accessible intercostal nerve on the involved side of the body. It is usually possible to check levels T2-12 in a slender individual. In an obese patient it may be impossible to go higher than T4 or T5 intercostal nerves. After testing intercostal conduction times, thoracic paraspinous muscles may be examined for denervation activity by standard electromyographic techniques (Nelson, 1967). The patient is then transferred to the surgical recovery room for postanaesthetic care.

\section{RESULTS}

In the five years following the development of intercostal conduction studies at Baylor 160 patients ( 54 male and 106 female) were evaluated for thoracic radiculopathy by this technique. The median age of these patients is 42 years with a range of 12-82 years. Nine hundred and seventy-one intercostal nerve conduction delays are recorded from these patients. The median conduction delay is $3.0 \mathrm{msec}$. The mean value for those intercostal delays considered normal is $3 \cdot 1 \mathrm{msec}$. The criteria for abnormality are:

1. Delay greater than $5.0 \mathrm{msec}$ with dispersion of evoked response

2. Delay greater than $6.0 \mathrm{msec}$

3. Delay $\mathbf{2} \cdot 0 \mathrm{msec}$ greater than the mean delay for that patient

An example illustrating number 3 above is as follows:

$\begin{array}{llll}\text { T5 } & 1.8 \mathrm{~ms} & \text { T9 } & 2 \cdot 1 \mathrm{~ms} \\ \text { T6 } & 2.0 \mathrm{~ms} & \text { T10 } & 4.8 \mathrm{~ms} \\ \text { T7 } & 2.1 \mathrm{~ms} & \text { T11 } & 2.0 \mathrm{~ms} \\ \text { T8 } & 2.0 \mathrm{~ms} & \text { T12 } & 2.0 \mathrm{~ms}\end{array}$

T10 intercostal nerve would be considered abnormal.

No deaths are reported after intercostal nerve conduction study. The only significant complication was post-study pneumothorax in 14 patients $(8.8 \%)$. Two of these patients required chest tubes, with the remaining patients having 10 $20 \%$ pneumothorax requiring only bed rest for resolution. Because of this complication, routine pre-study chest films are recommended and routine post-study chest films are necessary. The incidence of pneumothorax is higher than that recorded in intercostal nerve block (Moore and Bridenbaugh, 1962).

Opaque thoracic myelography was done on 87 patients. Thirteen $(15 \%)$ of these are recorded as positive. All patients with positive myelograms showed abnormal intercostal nerve conduction studies at the level of the defect. Four of the 13 patients declined surgery. Four patients with herniated thoracic intervertebral discs, two with metastatic carcinoma, one with arachnoid cyst, and one with neuroma had a negative myelogram with a positive conduction study.

SURGERY Eighty patients are recorded as having had 86 surgical procedures. Seventy-one patients went to surgery on the findings of intercostal nerve study alone with nine patients having both myelographic and intercostal conduction study abnormalities. The following 86 surgical procedures were carried out: posterior rhizotomy or modified posterior rhizotomy (80), biopsy of metastatic carcinoma (1), excision of thoracic disc (1), excision of meningioma (1), excision of arachnoid cyst (1), excision of neuroma (2).

The following 86 findings at surgery are recorded: scarring around root (19), osteophytic or other bony entrapment (16), protruding intervertebral disc (9), neuroma (3), microscopic degeneration (3), metastatic carcinoma (2), compression between transverse process and rib (2), arachnoid cyst (1), multiple myeloma (1), herpes zoster (demonstrated by electron microscopy) (1), intradural meningioma (1), neuralgia with no surgical or microscopic evidence of aetiology (28).

Of the 74 patients undergoing posterior rhizotomy $14(19 \%)$ poor results are recorded. The follow-up period ranges from one month to $1 \frac{1}{2}$ years. A poor result was manifested by continued pain at the previous location or new pain in an adjacent location. An evaluation of patients with poor results fails to reveal any pattern. However, one poor result occurred in a patient with multiple myeloma and one in a patient whose surgery was performed at a 
different level from that recorded as abnormal by intercostal conduction study.

The only death reported postoperatively occurred from bacterial endocarditis in a patient who had metastatic carcinoma. Morbidity included one patient with pericarditis, one with toxic hepatitis secondary to halothane, one with pulmonary embolus, and two with pneumonitis. One patient who had a thoracic disc excision developed weakness of the left leg.

\section{CONCLUSION}

Intercostal nerve conduction study is an accurate technique for the definitive diagnosis of thoracic radiculopathy and for the establishment of the level involved. The only significant complication of intercostal nerve conduction study is pneumothorax which occurred in $8.8 \%$ of 160 patients undergoing 971 separate intercostal nerve conduction studies. The median intercostal nerve conduction delay is $3.0 \mathrm{~ms}$. The mean is 3.1 ms with a standard deviation of $0.7 \mathrm{~ms}$. Abnormality is established by a delay greater than $5.0 \mathrm{~ms}$ with dispersion of evoked muscular response, a delay greater than $2.0 \mathrm{~ms}$ more than the mean delay for the intercostal nerves of that patient, or any delay greater than $6.0 \mathrm{~ms}$. $Z$

Posterior rhizotomy after localization of the level in thoracic radiculopathy produced relief $c$ from pain in $81 \%$ of operated patients. During the last year of this study, with improved selec- 0 tion of patients and continued experience in intercostal nerve conduction study technique, this experience has improved, with $91 \%$ of $\underset{\gtrless}{\gtrless}$ patients having relief from pain.

\section{REFERENCES}

Barthakur, A., and Harden, K. A. (1961). Entrapment neuropathy of intercostal nerve. A case report. Journal of $\vec{\nabla}$ National Medical Association, 53, 493-495.

Caldwell, J. W., Crane, C. R., and Boland, G. L. (1968). ڤి Determinations of intercostal motor conduction time in $\vec{A}$ diagnosis of nerve root compression. Archives of Physical Medicine, 49, 515-518.

Dick, E. (1961). Intercostal nerve nipping due to rib dislocation. New Zealand Medical Journal, 60, 576-577.

Moore, D. C., and Bridenbaugh, L. D. (1962). Pneumothorax. Its incidence following intercostal nerve block. Journal of the American Medical Association, 182, 1005-1008.

Nelson, J. W. (1967). Electromyographic examination of the erectores spinae in patients suspected of having nerve roa $\omega$ lesions. (Abstract.) Electroencephalography and Clinic 霖 Neurophysiology, 23, 391-392.

White, J. C. (1965). Posterior rhizotomy: a possible substitu民 for cordotomy in otherwise intractable neuralgias of the trunk and extremities of nonmalignant origin. Clinica Neurosurgery, 13, 20-41. 